neutrons in the range 2-20 MeV., will be submitted shortly for publication in the Proceedings of the Royal Society.

$$
\text { H. S. W. Massey }
$$$$
\text { TsI-MING HU }
$$

Mathematics Department,

University College, London. Aug. 21.

${ }^{1}$ Arnold, W. R., and Roberts, A., Phys. Rev., 71, 878 (1947).

'Kellog, J. M. B., Rabl, I. I., Zacharfas, J. R., and Ramsey, N. F., Phys. Rev., 57, 677 (1940).

S See Sachs, R. G., Phys. Rev., 72, 91 (1947) ; Primakoff, H., Phys. Rev. 72,118 (1947); and Breit, G., and Bloch, I.; 72, 135 (1947) for a discussion of the present position.

- Rarita, W., and Schwinger, J., Phys. Rev., 59, 436 (1941).

8 Bailey, C. E., Bennett, W. E., Bergstrahl, T., Nuckolls, R. G. Richards, H. T., and Williams, J. H., Phys. Rev., "70, 583 (1916),

- Hoisington, L. E., Share, S. S., and Breit, G., Phys. Rev., 56, 884 (1939).

"Swartholm, N., "The Binding Fnergles of the Lightest Atomic Nuclep" (Lund: Hakan Ohlsson, 1945).

- Rarita, W., and Present, R., Phys. Rev., 51, 788 (1937).

- See however, Gerjuoy, E., and Schwinger, J., Phys. Rev., 61, 138 (1942).

\section{Crystal Structures of Zinc and Magnesium Benzene Sulphonates}

A RECENT note by A. Hargreaves ${ }^{1}$, of the Physics Department, College of Technology, Manchester, summarized the information obtained from a projection, along the $b$-axis, of the electron density in the unit cell of zinc $p$-toluenesulphonate. An investigation of the structures of the closely related zinc and magnesium benzene sulphonates, begun by one of us (A. D. I. N.) at Manchester at the suggestion of Dr. Hargreaves and with his assistance, has now been completed in this Laboratory. The positions of all the atoms in the unit cells have been determined by Fourier syntheses of projections of the electron density along the $b$ - and $c$-axes. The crystals used in this analysis were twinned across the face (100), with the consequence that reflexions $(h 00)$ of the twin components overlapped completely, (h05) reflexions were superposed, and there was partial overlap of reflexions (h04) and (h06). Some reduction in the accuracy of the $b$-axis projection results from the necessity for applying a correction to the observed intensities of these overlapping reflexions.

As was expected, the structures of the benzene sulphonates prove to be essentially similar to that suggested for zine $p$-toluenesulphonate by Hargreaves from his partial determination of the structure by means of the single projection along the $b$-axis. The metal atoms are surrounded by regular octahedra of water molecules, and the metal-to-water distances are in accordance with accepted values of the atomic radii. The benzene ring is plane and the average distance between the carbon atoms is $1 \cdot 40 \mathrm{~A}$. The sulphur-oxygen bond distance in the sulphonate group is $1.41 \mathrm{~A}$., and the sulphur atom is linked to the benzene ring by a bond of length $1.89 \mathrm{~A}$. in the magnesium compound and $1.81 \mathrm{~A}$. in the zine compound, the difference probably representing experimental error. The distribution of bonds about the sulphur atom appears to be regular tetrahedral, the bond angles in both compounds being approximately $109^{\circ}$.

The analysis, and details of the structure, will be published elsewhere.

\section{J. M. BRoOMHEAD} A. D. T. NiCOL

Crystallographic Laboratory, Cavendish Laboratory, Cambridge. Aug. 26.

' Hargreaves, A., Nature, 168, 620 (1946).

\section{A Rapid Method of Analysis for Certain Surface-Active Agents}

THE fact that certain cationic surface-active agents, such as cetyl pyridinium bromide, form stoichiometric salts with anionic surface-active agents such as the alkyl sulphates, alkyl sulphonates and alkyl aryl sulphonates, has been used as the basis of two titrimetric methods of analysis of the latter materials. That of Hartley and Runnicles ${ }^{1}$ uses bromphenol blue as an indicator to detect when excess cation-active material has been added, while that of Preston ${ }^{2}$ uses a remarkable surface tension change to mark the end-point. Unfortunately, neither method of determining the equivalence point is completely satisfactory. In the first case the colour change is not sharp, and in the second the point at which the surface tension change occurs is affected by the presence of inorganic salts.

In this laboratory we have developed a new method of end-point detection which gives accurate and reproducible results even in the hands of relatively unskilled personnel and under a wide variety of conditions, and does not suffer from the above defects. The method is based on observations by $\mathrm{Jones}^{3}$. He showed that whereas the chloride of the basic dyestuff methylene blue is insoluble in chloroform, the alkyl sulphate and related salts are freely soluble and may be extracted quantitatively from aqueous solution by chloroform provided that the chain-length of the organic portion is sufficiently large. (This fact was made the basis of a colorimetric estimation.) However, the extraction is almost completely inhibited if the aqueous phase contains a quantity of cation-active agent more than equivalent to the anionic material present. This is because the colourless cetyl pyridinium alkyl sulphate is more soluble in chloroform and is extracted in preference to the methylene blue salt. The blue colour therefore remains in the upper aqueous layer.

In the new titrimetric method, $10 \mathrm{ml}$. of an alkyl sulphate or other similar surface-active agent (approximately $0.004 M$ ) is pipetted into a $250 \mathrm{ml}$. stoppered reagent bottle. $25 \mathrm{ml}$. of aqueous indicator solution containing 0.003 per cent methylene blue (B.P.), $1 \cdot 2$ per cent sulphuric acid and 5 per cent sodium sulphate are added, followed by $15 \mathrm{ml}$. chloroform. A solution of cetyl pyridinium bromide (approximately $0.004 M$ ) is then added from a burette. After each small addition of the latter the mixture is shaken. At first the blue colour concentrates in the chloroform layer, but as the titration proceeds thare is a slow transfer of colour to the water layer. Later the rate of transfer increases, and eventually the colour of the two layers is visually the same. This is found to be the equivalence point. On further addition the chloroform layer lightens in shade, and becomes colourless.

The fact that the point at which the two layers have the same colour is the equivalence point has been exhaustively confirmed by titrating solutions of some pure sodium alkyl sulphates and many other similar substances of known purity with a cetyl pyridinium bromide solution, the strength of which had been determined by precipitation of its dichromate salt with excess potassium dichromate ${ }^{4}$.

The end-point is surprisingly sharp and can be reproduced to $0.1 \mathrm{ml}$. after a little practice. It is not affected by the presence of excess inorganic salt, acids, moderate quantities of organic solvents, commonly occurring temperature variations, or reason- 